\title{
Inwieweit umfasst die medizinische Wissenschaft die medizinische Wirklichkeit?
}

\author{
H. G. Pauli ${ }^{a}$, K. L. White ${ }^{b}$, I. R. McWhinney ${ }^{c}$
}

\section{Vorbemerkung und Vorausschau}

a ehemaliger Chefarzt an der medizinischen Klinik, Gründer und Direktor des Instituts für Ausbildungs- und Examensforschung der medizinischen Fakultät der Universität Bern, Schweiz.

b ehemaliger Deputy Director for Health Sciences, the Rockefeller Foundation, New York, Gründer und Direktor der Health Care Organisation der Johns Hopkins Universität, Baltimore, ehemaliger Associate Professor of Internal Medicine an der University of North Carolina, Chapel Hill, NC, USA.

c ehemaliger Chefarzt und Professor für Family Medicine, Centre for Studies in Family Medicine, University of Western Ontario, London, Ontario, Canada.

1 Selbstverständlich muss dabei auch der Begriff Wissenschaft festgelegt werden. Wir meinen eine Wissenschaft, «die einen umgrenzten Gegenstandsbereich systematisch nach ihm angemessenen Methoden erforscht, ordnet und die Fülle der so gewonnenen Erkenntnisse auf umfassende Grundsätze zurückzuführen und aus ihnen zu erklären sucht» [1]. Im Zentrum steht dabei die Definition des Gegenstandsbereiches. Im Gegensatz zu umschriebenen Einzelwissenschaften kann dieser für die Medizin als Ganzes nicht eindeutig und einmalig festgelegt, sondern mit dem Vorsatz zur Vollständigkeit lediglich angestrebt werden. Pragmatischer ist die Frage, ob mit dem zeitgeistigen Begriff Medizin sämtliche heute zugänglichen gesundheits- und krankheitsrelevanten Aspekte der Humanwissenschaften miteinbezogen sind. Dieser Beitrag geht von einer Verneinung dieser Frage aus.

Korrespondenz:

Prof. Dr. med. Hannes G. Pauli Oranienburgstrasse 13

CH-3013 Bern
Der Begriff «Medizin» steht für mehr als für den ärztlichen Arbeitsbereich. Andere Einrichtungen und andere Gesundheitsberufe sind für die Gesundheit vielleicht bedeutsamer als der ärztliche. Trotzdem soll hier, auf der konzeptionellen Ebene, vom Status Quo der ärztlichen Wissenschaft ausgegangen werden. Ihr Denkgebäude nimmt fast im gesamten Gesundheitswesen unserer szientistischen westlichen Gesellschaft eine dominierende Rolle ein. Die Autoren beziehen sich hier auf die zurzeit in etablierten akademischen Institutionen (vorwiegend Medizinische Fakultäten im westlichen Kulturbereich) dominierende Wissenschaft ${ }^{1}$. Es geht nicht um deren Ablehnung, sondern um ihre Erweiterung durch historisch längst anerkannte philosophische Ansätze. Diese Erweiterung muss heute als Konsequenz der Erkenntnisse der Physik des 20. Jahrhunderts als wissenschaftlich bezeichnet werden. Am etablierten Wissenschaftsbetrieb Unbeteiligte mögen in ihr aber ebensogut eine von einem übernommenen Fundamentalismus (oder Glaubenssystem) befreite Zuwendung zu alltäglichen, aber essentiellen Selbstverständlichkeiten sehen.

Ausgangspunkt ist die Frage: Was ist der Mensch in einer wissenschaftlichen Sicht, die zunächst durch keine historisch vorgegebenen Modelle eingeschränkt ist? Mit der Aufklärung hat sich die Antwort auf diese Frage von einer vorwiegend mystischen und spirituellen Ebene auf eine solche von materiellen Strukturen und Funktionen, auf Descartes räumlich ausgedehnte «res extensa», verlegt. Diesem «Ding» waren u.U. immaterielle Elemente («res cogitans») beigegeben, die in der Folge gegenüber den materiellmechanistischen Anteilen laufend an Gewicht verloren. Dieses Prinzip I ging und geht von der Metapher einer Maschine (Descartes Uhrwerk) aus. Als geschlossenes System von physikalischen und chemischen Abläufen wird es aufgrund von «Naturgesetzen» als «rational» (nach der gängigen Definition = «wissenschaftlich») bezeichnet. Es kann durch Eingriffe von ebenfalls naturgesetzlicher Art beeinflusst, verändert werden. Diesem Denkmodell hat die heutige
Medizin ihre unzähligen und beeindruckenden Machbarkeiten zu verdanken.

Ein «Systemisches» (u.a. über das Materielle hinausgehendes) Denkmodell (Prinzip II) erlaubt die Integration von bislang ausgegrenzten, spezifisch humanen Anteilen. In Abbildung 1 ist ein solches Modell, modifiziert nach Jakob und Thure von Uexkülls [2, 3] «Funktions- bzw. Situationskreisen», graphisch dargestellt. Das Lebewesen/Individuum ist zur Aufrechterhaltung des Lebens auf die Wahrnehmung seiner Umgebung angewiesen (1). Es interpretiert diese Umgebung und reagiert darauf in einer individuellen, von aussen nicht unbedingt einsehbaren Weise («Selbstorganisation», s.u.) (2). Die Auswirkung der damit veränderten Umgebung (3) kann auf das Individuum zurückwirken und von diesem wahrgenommen werden. Ein Problem, das aus der ersten Wahrnehmung (1) hervorging, ist damit u.U. gelöst. Falls nicht, kann es auf dem skizzierten Kreis zu einer positiven oder negativen zirkulären Rückkoppelung (Interaktion) kommen, bis das Verhältnis zwischen Individuum und Umgebung ein Gleichgewicht findet. Die Veränderung in der Umgebung kann sich aber auch anderswohin (3) (Pfeil nach oben) auswirken, d.h. das Individuum nimmt nicht wahr, welche Wirkung es auf die Umgebung hat.

In Abbildung 2 sind einige Beispiele von solchen Funktionskreisen $\mathrm{zu}$ einem IndividuumUmgebungssystem zusammengebaut. Auf komplexer werdenden «Ebenen» dieses Schemas von physikalisch-chemisch-technisch über somatisch und psychisch bis soziokulturell - entstehen («emergieren») neue Eigenschaften, die sich durch diejenigen weiter «unten» nicht erklären lassen. Diese Emergenz von «unten nach oben» ist in der Grafik durch den zunehmenden Umfang des Zylinders «Individuum» angedeutet $^{2}$. So wird ein Nahrungsmittel (Brot) erkannt, es wird aufgenommen und dient dem Aufbau, der Existenz auf der somatischen Ebene (Anabolismus). Gefahren (Gift) werden erkannt und gemieden. Der Fötus/das Kind überlebt und entwickelt sich dank der somatischen und soziokulturellen Verbindung mit der Mutter. Heilungsprozesse werden u. a. durch einen Austausch zwischen Individuum und Arzt ermög- 


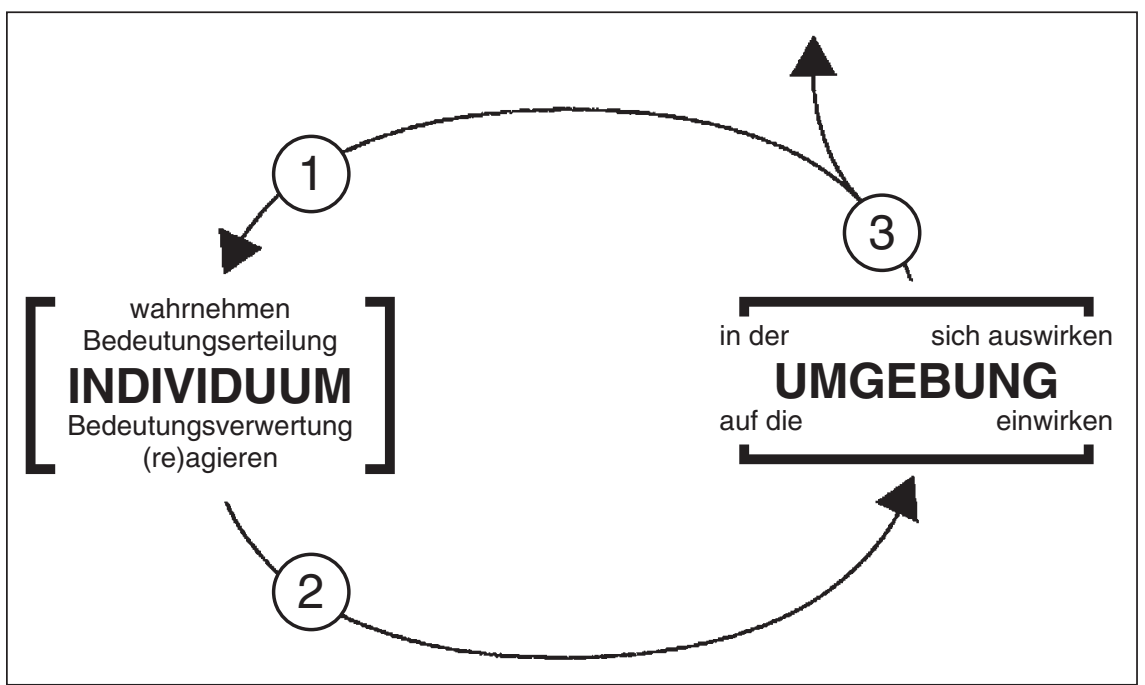

2 Die perspektivische Darstellung darf nicht im Sinne einer räumlichen Struktur gedeutet werden. Sie dient lediglich einer geeigneten Anordnung von hier exemplarisch ausgewählten wesentlichen Abläufen. Das wichtigste sind somit die verbindenden Pfeile, welche die für das Leben notwendigen Prozesse (s. Abb. 1) repräsentieren. licht. Diesen Aussenbeziehungen sind, in der Abbildung nicht speziell gezeigt, intern im Organismus solche auf Mikroebenen - z. B. der biochemischen Ebene - beizufügen. Zellen nehmen die Anwesenheit eines Hormons in der umgebenden Körperflüssigkeit wahr, dieses wird angedockt und veranlasst Entwicklungsprozesse im Zellinneren (Wachstum, Zellteilung u.a.). Schliesslich verbinden vertikal-zirkuläre physikalische, chemische und immaterielle Interaktionen (in Abb. 2 rechts als Pfeile zusammengefasst) die Ebenen des Systems miteinander, von «physikalisch-chemisch» über «somatisch» und «psychisch» bis «soziokulturell» («aufwärts») und zurück («abwärts»). Zusammenfassend sind damit Abläufe von Gesundheit skizziert; bei fehlenden oder fehlerhaften Wahrnehmungen bzw. fehlerhaften Bedeutungserteilungen diejenigen von Krankheit.

Das hier vorgestellte zweite Modell ist nicht neu. Es entspricht der Denkweise einer Minderheit von wissenschaftlich und forschend Tätigen. Vielleicht häufiger haben sich Termini («systemisch», «Paradigmenwechsel» u.a.) eingenistet, unter Beibehaltung traditioneller Denk- und Arbeitsweisen nach Prinzip II einherzugehen. Das Modell beansprucht auch keine einmalige Gültigkeit; andere mögen unseren Zielen näherkommen. In der Zukunft werden solche zweifellos in den Vordergrund treten [4]. Anlass für diesen Beitrag ist die globale Konsternation über die Unzulänglichkeiten der Gesundheitsversorgung, die paradoxerweise mit dem phänomenalen Fortschritt in der biomedizinischen Forschung und Technologie einhergeht.
Wie lässt sich wissenschaftlich einordnen, was wir beobachten?

An den Anfang eines Hinterfragens der skizzierten Denkmodelle sollen nicht eine Theorie oder Regel, sondern zwei konstruierte Fallbeispiele gestellt werden:

\section{Fallbeispiel I}

(Situation: In Westberlin domiziliertes Ehepaar im Jahre 1962): Der Ehemann wurde 1961 während eines Besuches bei seinen Eltern in der Ostzone vom Mauerbau überrascht und zurückgehalten. Monatelange Vorbereitungen lassen den Plan einer Flucht durch den Todesstreifen reifen. Auf verschlungenen Wegen erhält die Frau davon Nachricht. Nach dem Gelingen dieses Unternehmens in einer vorbestimmten Nacht, so lautet die Botschaft, wird sich der Mann telefonisch bei seiner Frau melden.

In der bange erwarteten Nacht ist die erschöpfte Frau schliesslich eingeschlafen. Um 3 Uhr schrillt das Telefon ...

\section{Fallbeispiel II}

(Die gleichen Personen in einer anderen Situation): Die Frau kehrt um Mitternacht aus dem Krankenhaus zurück, in dem ihr Ehemann in einem kritischen Schockzustand nach ausgedehntem Myokardinfarkt auf der Intensivpflegestation liegt. Sie hat dort über 24 Stunden bange wartend verbracht, während sich der Zustand des Patienten zwar leicht gebessert hat, aber kritisch geblieben ist. Schliesslich haben ihr die Ärzte empfohlen, sich zu Hause für einige Zeit auszuruhen. Man werde weiterhin alle möglichen Massnahmen zur Wiederbelebung fortführen. Sie solle ruhig schlafen; keine Nachricht bedeute gute Nachricht.

Die Frau legt sich zu Hause nieder und schläft tief ein. Um 3 Uhr schrillt das Telefon ...

In beiden Fällen löst eine bezüglich materiell beschreibbarer Umstände identische Situation bei der Frau intensive somatische und psychische Reaktionen aus. Zunächst treten relativ unspezifische, mit einer Adrenalinausschüttung verbundene Symptome auf, u.a. eine Beschleunigung von Herzrhythmus und Atmung. Der weitere Verlauf wird in Situation I und II je unterschiedlich sein, im ersten Fall begleitet von einer Aufhellung der Stimmung und Freude, im zweiten von Angst, psychischer Dämpfung, Depression und Verzweiflung. Für beide Fälle können je unterschiedliche neurale Abläufe und 


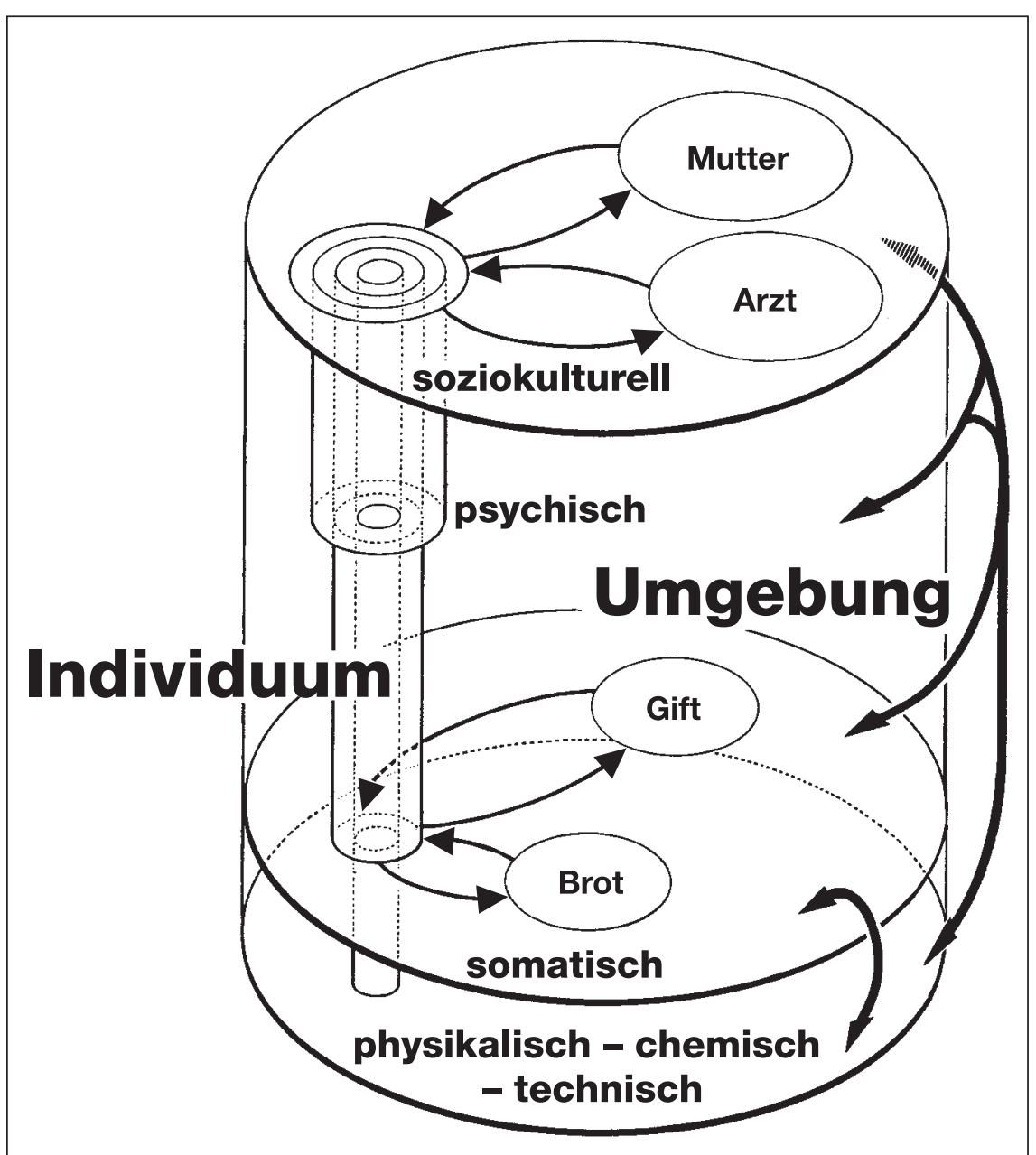

Konstellationen von Neurotransmittersubstanzen angenommen werden. Desgleichen lassen sich unterschiedliche klinische Befunde erheben, körperliche Manifestationen eines Wohlbefindens einerseits und Funktionsstörungen verschiedener Organe anderseits.

Wie ist so etwas möglich? Wie kann dieses physikalische Phänomen, das Klingeln des Telefons, zwei dermassen unterschiedliche Bedeutungen haben für je einen vergleichbaren Organismus, und in zwei unterschiedlichen Lebenssituationen dermassen unterschiedliche emotionale und somatische Reaktionen auslösen? Anders ausgedrückt, wie kann je ein unterschiedlicher immaterieller und interpretierender Zustand der Psyche, je eine «res cogitans» mit einer gleichen materiell «Raum beanspruchenden res extensa» des Körpers «abwärts» (s. Abb. 2), kausal interagieren und damit am Anfang unterschiedlicher psychobiologischer Abläufe stehen? Dominiert hier Bedeutung Chemie? Diese Frage überfordert die Erklärungsbasis der von unserer Kultur anerkannten Medizin. Im heute mehr- heitlich etablierten Vokabular kann die Frage nicht beantwortet werden.

\section{Eine kurze Geschichte des Reduktionismus}

Mit der zunehmenden, dem Prinzip I entsprechenden Aufteilung der westlichen medizinischen Wissenschaft in Physiologie, Biochemie, Mikrobiologie, Immunologie, Molekurlarbiologie und Genetik wurden Erklärungen für die Phänomene im lebenden oder toten Körper gefunden. In Analogie zu dieser Fächerbildung war dabei der dominierende methodische Ansatz die Analyse, das Zerlegen einer Struktur oder eines Ablaufs in ihre Komponenten («Teile» s.u.). Aufgrund dieser Entwicklung haben prominente Vertreter dieser sich neu formierenden medizinischen Wissenschaften eine entscheidende Rolle bei der Aufwertung ihrer wissenschaftlichen Konzepte zu einer Weltanschauung gespielt. Das analytische Prinzip wurde zur Voraussetzung, zur Verpflichtung des Wissenschaftlers [5]. Diese Dominanz des Newton'schen Paradigma blieb bis heute bestehen. Der Nobelpreisträger Arthur Kornberg (*1918), unter vielen anderen, bestätigt diese Sichtweise eines intimen Kreises zeitgenössischer Wissenschaftler, wenn er fordert, dass wir eine «reduktionistische Sichtweise, die auch die meine ist, [...] rückhaltlos anerkennen", nämlich «dass sowohl Form als auch Struktur des Gehirns und des Nervensystems nichts anderes ist als Chemie, [...] dass der Geist als Bestandteil des Lebens Materie ist, nichts anderes als Materie.»... Es sei abwegig, meint Kornberg, «die Vorstellung aufrechtzuerhalten, dass menschliches Verhalten nicht durch Chemie und die physikalischen Gesetze erklärt werden kann» [6]. Aus der Vielfalt der eingangs erwähnten Fächer erwachsen somit die nun als Grundlagen bezeichneten Teile (Zellen, Moleküle u.a.), aus denen («aufwärts», s. Abb. 2) der menschliche Organismus hervorgegangen sei. Im Rahmen dieses summativen Menschenbildes werden psychologische und soziale Phänomene in den vom «biomedizinischen» Zentralbereich weitgehend abgesetzten Disziplinen Psychologie und Soziologie behandelt und im besten Fall mit restriktiven Begriffen wie «Risikofaktoren» charakterisiert. Eine derartige methodologisch begründete Dominanz des Materiellen im Kontext des wissenschaftlichen Paradigmas des 19. Jahrhunderts hat zu einer Unterscheidung zwischen präziser und nützlicher «harter» und unpräziser und nicht nützlicher «weicher» Wissenschaft geführt. Solche Sichtweisen führen zu Abstraktionen, welche die Wahrnehmung von Phänomenen generalisieren, organisieren, u.U. transfor- 
mieren und zu einem beträchtlichen Anteil vereinfachen.

Eine zentrale Abstraktion ist das Konzept der Diagnose, wie es in der International Classification of Diseases [7] festgelegt ist. Damit wird uns die Auffassung vermittelt, dass die Diagnose die einzig mögliche philosophische Basis für die Formulierung und Organisation unserer Daten darstellt. Dem ist entgegenzuhalten, dass erkrankte Personen vielmehr als «Krankheiten» Realitäten sind. Der Begriff Diagnose bedeutet weniger eine Umschreibung von Fakten als von etablierten medizinischen Konventionen - er ist einem «Glaubenssystem» zugeordnet. Als solcher ist er ein Arbeitsinstrument, dessen Benutzung, Nichtbenutzung oder alternative Benutzung zu unterschiedlichen Handlungen und Folgen führt. So lenkt beispielsweise der diagnostische Begriff von entweder Angina pectoris oder koronare Herzerkrankung in einer identischen Situation die Aufmerksamkeit der Diagnostizierenden in unterschiedliche Richtungen. Im ersten Fall findet voraussichtlich vor allem eine Orientierung an den subjektiven Wahrnehmungen des Patienten und an der Person als Ganzes statt. Im zweiten Fall steht der für die Gefässe und das Herz bedeutsame Mechanismus im Vordergrund. Man mag einwenden, dass erfahrene Angehörige der Gesundheitsberufe kaum ihre Sicht ändern werden, indem sie einen Begriff für einen andern setzen. Gegenüber diesem berechtigten Argument muss die Formierung einer paradigmatischen Sicht von Krankheit und Medizin bereits ab Beginn des Medizinstudiums und die Tendenz ihrer Fixierung und Verhärtung während der Ausübung des Berufes betont werden. Wir haben es in dieser Analyse zu unserer Aufgabe gemacht, die Verwendbarkeit der etablierten Sichtweisen $\mathrm{zu}$ evaluieren und alternative oder erweiterte Modalitäten des Denkens über erkrankte Individuen, Krankheiten, Medizin, Gesundheit und deren praktische Implikationen für unser Gesundheitswesen zu ermitteln.

Parallel zur Etablierung eines damit skizzierten «biomechanischen» Modells waren seit Descartes Zeiten Philosophen, Naturbetrachter, Dichter, Künstler und engagierte Ärzte bewusst oder unbewusst vertraut mit über den Bereich der «Naturgesetze» hinausgehenden Umständen von Gesundheit und Krankheit. Dieser «zweite Pfad» der Entwicklung von Weltanschauungen weist u.a. vom Mathematiker, Physiker und Philosoph Blaise Pascal (1623-1882) zu Lord Shaftsbury (1671-1713), Jean-Jacques Rousseau (1712-1778) und später zu den Ärzten und Philosophen der deutschen romantischen Epoche im 19. Jahrhundert [8].
All diesen historischen Ansätzen, die sich unter anderem zum Ziel setzten, Informationen über das Leben und Erleben der Menschen zu sammeln, die mit Gesundheit und Krankheit zu tun haben, fehlte eine wissenschaftliche Grundlage im heute gebräuchlichen Sinn. Diese mehr intuitiven Erkenntnisse wurden immer wieder verdrängt durch die als überzeugender empfundenen Thesen der Aufklärung (vgl. Prinzip I in der Vorbemerkung) und der post-Newtonschen Ära.

\section{Neuorientierung}

Die grundsätzlichste, diesmal wissenschaftliche Neuorientierung des Denkmodells aus dem 17. Jahrhundert erfolgte im 20. Jahrhundert mit der Einführung der Quantenmechanik durch Niels Bohr (1885-1962) [9], Erwin Schrödinger (1887-1961), Werner Heisenberg (1901-1979) sowie der Relativitätstheorie durch Albert Einstein (1879-1955). Die neue Sichtweise (vgl. Prinzip II in der Vorbemerkung) wurde im weiteren Verlauf konkretisiert durch die Ansätze der Cybernetik von Claude Shannon (*1916) und der Systemtheorie von Ludwig von Bertalanffy (1901-1977) [10] sowie der Ungleichgewichtsthermodynamik und des Phänomens der Ordnungsbildung aus dem Chaos durch Ilya Prigogine (1917-1984) [11]. Drei hauptsächliche Elemente charakterisieren diese historischen wissenschaftlichen Annahmen und Konzepte:

Erstens kann wissenschaftliche Wahrnehmung - so wie Wahrnehmung ganz allgemein nicht mehr als «objektive» Projektion einer «Realität» oder einer «Wahrheit» in das Bewusstsein des Beobachters, des Forschers betrachtet werden. Sein individuelles Bewusstsein bestimmt die Wahrnehmung mit [12]. Wenn auch viele einfache, alltägliche Beobachtungen und Messungen nicht wesentlich durch die Untrennbarkeit von Beobachter und Beobachtetem betroffen werden mögen, kann das «Subjekt» komplexere «Objekte» bedeutend verändern. Diese gegenseitige Veränderung während der Kommunikation zwischen zwei Subjekten (wobei jedes Subjekt Objekt des anderen ist) spielt in der Ärztin-Patient-Kommunikation eine besonders bedeutsame Rolle. Körpersprache, erotische Signale und Erröten beispielsweise beeinflussen die Interpretation und die Bedeutungserteilung gegenseitig. Der «Plazeboeffekt» ist ein weiteres Beispiel für dieses Phänomen.

Zweitens müssen sowohl die belebten als auch die unbelebten Komponenten der Umgebung als Systeme gesehen werden. Sie sind in die 
individuelle Wahrnehmung integriert, als Teil eines weiterreichenden Suprasystems einerseits und als integrierender Zusammenschluss von Subsystemen anderseits. Eigenschaften, die aus der Verbindung von Subsystemen hervorgehen (Emergenzen), lassen sich nicht durch die Summe der Eigenschaften ihrer Subsysteme erklären bzw. voraussagen [13].

Drittens ist Selbstorganisation das zentrale Phänomen, das neuen (emergierenden) Qualitäten, speziell von Biosystemen, zugrundeliegt, die während ihrer Phylo- und Ontogenese auftreten [14].

Während das Newton'sche Paradigma immer weniger die erhobenen Daten zu erklären vermochte, verdichteten sich diese Erkenntnisse zu zusätzlichen Voraussetzungen und Kriterien wissenschaftlicher Arbeit. Bemerkenswert ist dabei der Umstand, dass diese Erkenntnisse und Konzepte und deren zunehmende Akzeptanz aus dem Bereich der Physik stammen, dem Schlüsselfachbereich der «modernen» materialistisch orientierten Wissenschaft, insbesondere auch der Medizin. Mehrere alte intuitive Erfahrungen wurden damit auf die Ebene der wissenschaftlichen Erklärung gehoben.

Die Konzepte Kausalität, Biosemiotik, Geistkörper und Salutogenese sollen in der Folge als Beispiele dienen für die Richtung, in der sich ein neues Paradigma für das 21. Jahrhundert entwickeln könnte.

\section{Kausalität}

Die Grenzen der biomechanischen Kategorie des Denkens werden deutlich gemacht durch den scheinbar trivialen Umstand, dass die Leiche nicht identisch ist mit dem lebenden Körper. Der letztere unterscheidet sich vom Leichnam durch seine inhärente Autonomie und seine Fähigkeit, sich selbst zu organisieren. Der Leichnam mag auf einen physikalischen oder chemischen Stimulus in einer spezifisch mechanischen und voraussagbaren Art reagieren. Der lebende Organismus reagiert nicht nur auf derartige, sondern ebenfalls auf psychologische Stimuli als Ganzes, indem er seinen Prozess der Selbstorganisation verändert (adaptiert). Die Bedeutung dieser externen «Stimuli» (die Verwendung dieses Begriffes ist in diesem Kontext fragwürdig) muss relativiert werden. Humberto Maturana ( $\left.{ }^{\star} 1928\right)$ und Francisco Varela (*1946) beschreiben sie als «Perturbationen». Die damit zusammenhängenden, aber davon nicht unbedingt logisch ableitbaren Prozesse machen Organismen und Individuen zu Systemen, die «ihre eigene Welt hervorbringen», m.a.W. ein individuelles Bewusstsein entwickeln [13].

Hier mag eingewendet werden, dass die mo- lekulare und die genetische Biologie im Begriffe sind, viele Strukturen und Funktionen des Lebensprozesses und deren Störungen zu identifizieren. Ohne die auf diesen Gebieten erzielten faszinierenden Einsichten in Frage zu stellen, muss betont werden, dass molekulare und genetische Komponenten nicht Botschaften (Zeichen und Information), sondern die chemischen Träger von Botschaften (d.h. Botschafter) sind, welche den Lebensprozess in Gang halten. Auch die Interaktion von Transmittersubstanzen mit den Rezeptoren auf den Zelloberflächen oder der Fluss über neurale und transmembrane Spannungsdifferenzen erlauben keine Schlussfolgerungen bezüglich des Inhalts (der Bedeutung) von Information (Zeichen oder Botschaft). Es sind lediglich Transmissionsphänomene, deren Bedeutung für den allgemeinen Lebensprozess verborgen bleibt. Als mechanistische Metapher formuliert, besteht zwischen Radiowellen, -sender oder -empfänger (= Träger der Signale) und der Musik, die zu hören ist (= entschlüsselte Signale), ein kategorialer Unterschied. Ausserdem sind die Verbindungen zwischen beobachteten molekularen und genetischen Komponenten auf der einen Seite und der Struktur und Funktion des ganzen Organismus auf der andern alles andere als einfach und linear. Bezüglich des Genoms hat die Nobelpreisträgerin Barbara McClintock (*1902) beeindruckend seine Aktivität und insbesondere seine Reaktionen auf eine grosse Zahl von Einwirkungen, die aus dem Inneren und der Umgebung des Organismus stammen, beschrieben. In ihren Worten ist das Genom «ein hoch sensitives Organ der Zelle, das die ganze genomische Aktivität überwacht und üblicherweise auftretende Fehler korrigiert, ungewöhnliche und unerwartete Ereignisse registriert und darauf reagiert, oft indem es sich selbst rekonstruiert. Wir kennen die Komponenten des Genoms, welche einer solchen Restrukturierung zugänglich sind. Wir wissen aber nicht, auf welche Art die Zelle Gefahren registriert und darauf antwortet, in einer Weise, die oft bemerkenswert ist» $[15] .{ }^{3} \mathrm{Ge}$ netischer Ausdruck (die Transformation genetischer Botschaften in Strukturen und Funktionen des biologischen Systems) erweist sich demzufolge als hochkomplexer Prozess, an dem auch «epigenetische» Elemente beteiligt sind. Das ganze ist inkompatibel mit striktem genetischem Determinismus, dem Anlass zu grandiosen Hoffnungen und Voraussagen einer prophetisch orientierten Genetik. Der Molekularbiologe Richard Strohman (*1927) teilt diese Hoffnungen ebenfalls nicht. In seinen Worten ist die eng definierte und einwandfreie Theorie des Gens der «Watson-Crick-Ära» fälschlicherweise in eine 
Theorie und ein Paradigma des Lebens umgedeutet worden: d.h. in eine «wiederbelebte und exklusiv molekulare Form eines genetischen Determinismus» [16]. Selbst einer der Aufklärer der biochemischen Identität des menschlichen Genoms, Craig Venter, warnt vor Schlüssen von den Teilen auf das Ganze: «Zwei Trugschlüsse gilt es auszuräumen: Determinismus, die Meinung, sämtliche Charakteristika einer Person seien durch das Genom «hart verdrahtet), und Reduktionismus, die Annahme, mit der Kenntnis der menschlichen Genomsequenz sei es lediglich eine Frage der Zeit, bis unser Verständnis der Genfunktion und -Interaktion eine vollständige Beschreibung der menschlichen Variabilität möglich macht» [17].

\section{Biosemiotik}

Mit der Beschränkung der Wahrnehmung auf physikalische oder biochemische Lebensphänomene und der Verwechslung ihrer Rolle als Kommunikation statt als Träger von Kommunikation limitiert die heutige Wissenschaft in schwerwiegender Weise das Verständnis von Krankheit und noch mehr dasjenige von Gesundheit (siehe unten). Einfachere und überzeugendere Modelle werden entstehen, wenn der Informationsfluss initial mehr Beachtung findet als seine exklusiv materiellen Elemente, d.h. Strukturen und Signale. Wissenschaftliches Verständnis impliziert damit die Notwendigkeit biosemiotischen Denkens, d.h. die Interpretation von Signalen und das Erkennen von deren $\mathrm{Be}$ deutung [18]. Nochmals sei an die mechanische Metapher Radiosender und -empfänger einerseits und das Hören von Musik erinnert.

Die obigen, auf Kausalität und Semiotik bezogenen Argumente interpretieren Gesundheit und Krankheit als nicht nur durch unsere materiellen Umstände (z.B. Gene und Erreger) hervorgerufen, sondern ebenfalls durch unsere Lebenssituation und die Bedeutungen, die wir diesen und anderen, auch nicht materiellen Elementen zuschreiben. Schon die Verwendung von Begriffen manifestiert und konsolidiert demzufolge das heutige Ungleichgewicht des Konzeptes «Leben». Das Hinzufügen der Wurzel «bio» zu den Begriffen der Naturwissenschaften, Medizin und Psychiatrie übersetzt sie in «Biologie», «Biomedizin» und «Biopsychiatrie», was paradoxerweise deren materielle (z. B. biochemische, molekulare) Elemente, unter Ausschluss oder mindestens Beschränkung ihrer nicht materiellen Bedeutungen, betont [19].

Die integrativen Elemente lassen sich unter 4 Mindbody, im Englischen eingeführt, eigentlich das GeistKörper-Umgebungssystem. dem aufwendigen Begriff "psychosomatosoziosemiotisch» zusammenfassen. Das integrierende
Modell versucht zu erklären, warum in einem lebenden, sich selbst organisierenden System Informationen wesentliche Regulatoren biologischer Prozesse sind. Informationen aktivieren systemische Rezeptoren, die sie in Botschaften verwandeln, welche physiologische Veränderungen initiieren. In dieser Weise sind durch unser System geleitete Botschaften selbst ätiologische Faktoren. D.h., Anschauung formiert Biologie. Lebensprozesse überschreiten die Grenzen des «Körpers» mittels Botschaften oder Zeichen. Anstelle des obsolet gewordenen Begriffes «Biomedizin» bietet sich «Infomedizin» an $[5,20]$.

\section{Geistkörper}

Der Körper wird damit im Konzept des lebenden Organismus ein wissenschaftlich fragwürdiges Konzept. Geistkörper ${ }^{4}$ erscheint damit der realistischste Rahmen für die Untersuchung von Lebensprozessen zu sein. Es geht um nichts weniger als um eine Aufhebung der Trennung zwischen Körper und Seele/Geist und damit zwischen Natur- und Geisteswissenschaft, an der in der heutigen Medizin krampfhaft festgehalten wird [21]. Im Vordergrund steht weniger die Gültigkeit des heutigen medizinischen Paradigmas, als dessen Unvermögen, all die erhältlichen Informationen mit einzubeziehen, die auf die Gesundheit jedes einzelnen Patienten einwirken.

\section{Salutogenese}

Ein letzter grundsätzlicher Vorbehalt gegenüber den zentralen Konzepten der etablierten medizinischen Wissenschaften mit ihrer prädominierenden Beschäftigung mit Krankheit hat mit der Produktion von Gesundheit zu tun. Die Formulierung «Produktion von Gesundheit» ist eine im Rahmen des heutigen medizinischen Denkens unübliche Umschreibung. Gesundheit wird vor allem als unhinterfragte, präexistierende, statistische Norm betrachtet, als Selektion von physikalischen und chemischen Nominalwerten, welche bei Krankheiten häufig über- oder unterschritten werden. Auf der Basis des in der Vorbemerkung skizzierten empirisch fundierten Funktionskreises (Abb. 1) erscheint die Produktion von Gesundheit für das Individuum-Umgebungssystem als ausserordentlich fruchtbare Vorstellung. Sie führt das postbiomechanische Konzept von Bedeutung $[2,22]$ ein.

Der Medizinsoziologe Aaron Antonovsky (1923-1994) ist parallel zu und unabhängig von den oben erwähnten Einsichten dieser Denkweise gefolgt [23]. Er war einer der ersten Kritiker der fast exklusiven Faszination der heutigen medizinischen Wissenschaftler durch die Patho-

genese. In der Absicht einer Bearbeitung bisher 
unberücksichtigter Bereiche der medizinischen Wissenschaften, die sich mit der Produktion und der Erhaltung von Gesundheit befassen, hat er einen neuen Terminus vorgeschlagen: Salutogenese (das Entstehen von Gesundheit). Antonovsky hat die drei zentralen Voraussetzungen für salutogenetische Phänomene und Prozesse in seinem Konzept des Kohärenzsinnes zusammengefasst. Dessen hauptsächliche Qualitäten sind die Verständlichkeit, die Beeinflussbarkeit und der Bedeutungsgehalt der Umgebung, die eine Person - oder generell ein Lebenssystem - als ihr eigenes empfindet. Indem Erteilung und Nutzung von Bedeutung eine Vorbedingung für derartige Sicht- und Empfindungsweisen sind, stimmt das salutogenetische Modell von Antonovsky mit den erwähnten zirkulären Modellen (siehe Abb. 1) Jakob von Uexkülls (1865-1944), und Thure von Uexkülls ( $\left.{ }^{\star} 1908\right)$ überein. Sowohl der Kohärenzsinn als auch die Prozesse im Funktions- und Situationskreis wurden hier der Ebene des menschlichen Bewusstseins zugeordnet. Im biochemischen bzw. molekularbiologischen Bereich lassen sich jedoch analoge, «isomorphe» Abläufe beobachten (s. die Erkenntnisse von Barbara McClintock, inkl. Fussnote auf Seite 2637). Menschlich-psychische Funktionen lassen sich damit als späte und differenzierte Stufe einer biologischen Evolution verstehen.

Obwohl derartige systemische Sichten durch empirische Daten belegt sind [23, 24], ist ihre Akzeptanz durch medizinische Wissenschaftler marginal geblieben. Selbstverständlich muss und wird sich die pathogenetische Analyse und deren Auswertung zuhanden der Gesundheitsversorgung weiterentwickeln. Die Auswirkungen des nahezu ausschliesslich pathogenetischen Gedankenmodells in Form eines gigantischen Komplexes akademisch-wissenschaftlichen Wissens und - damit eng verbunden - einer entsprechenden industriell-kommerziellen Produktion haben sich aber schwergewichtig auf ärztliches Denken und praktisch-medizinische Erfahrung niedergeschlagen. Die Suche nach ergänzenden Sichtweisen ist demnach Voraussetzung für eine Neuorientierung ärztlichen Handelns.

Mit einem erneuten Interesse an der Produktion und der Erhaltung von Gesundheit muss die Aufmerksamkeit der medizinischen Berufsangehörigen zunehmend auf das gerichtet werden, was heute in der Alltagssprache eine gesunde Person genannt wird. Eine Person, im Gegensatz zum Patienten - der bislang im medizinisch-fachlichen Kontext vorgezogene Terminus -, sollte imstande sein, die traditionelle Asymmetrie zwischen ihr und dem meist dominierenden Arzt auszugleichen. In diesem Sinne muss auch der letztere die Rolle als Person einnehmen, womit die «objektive» Beratung zum subjektiven Erleben wird [25]. Dieses Miterleben kann quasi als «Instrument» für die Herstellung einer «gemeinsamen Wirklichkeit» $[3,26]$ mit der betreuten Person gesehen werden.

Als Konsequenz eines erweiterten und gesundheitsorientierten medizinischen Modells kann Krankheit und Kranksein somit unter anderem als Unfähigkeit einer Person oder eines Organismus interpretiert werden, Bedeutung aus der internen und externen Umwelt zu generieren und deren Möglichkeiten und Angebote zu nutzen.

Zusammengefasst gestattet eine intensive Beschäftigung mit den Konzepten von Kausalität, von Biosemiotik und von Geistkörper sowie der Produktion von Gesundheit, eine historisch gewachsene phänomenologische Sicht von Lebenssystemen auf die Ebene wissenschaftlicher Erklärbarkeit zu heben.

\section{Wissenschaftliche Grundlagen}

Ein neues Denksystem erfordert eine Revision seiner konstituierenden Begriffe. Der Ausdruck «Grundlagenwissenschaften» mag als Beispiel dienen. Er umschreibt in medizinischen Ausbildungs- und Forschungsinstitutionen die Basis, die all den vorwiegend anwendungsorientierten Wissenschaften und Handlungsbereichen zugrundeliegt. Sie erstrecken sich von der geschichtsträchtigen Anatomie und Physiologie zu vor kürzerer Zeit eingeführten Bereichen wie Molekularbiologie und Biophysik. Typischerweise befassen sich diese Disziplinen viel mehr mit Teilen - Organen, Zellen, Molekülen - sowie deren womöglich mathematisch beschreibbaren Funktion - als mit Organismen, Personen oder gar einem Individuum-Umgebungskontext.

Im Anschluss an unsere Analyse des etablierten medizinischen Paradigmas müssen wir uns überlegen, ob diese Teile tatsächlich grundlegend sind. Zweifellos sind sie wichtig; wir benötigen viele dieser Wissensgebiete, wenn wir Medizin praktizieren, um Individuen und Populationen zu helfen. Zukünftige Kliniker und Klinikerinnen können allerdings nicht mehr zurechtkommen mit einer ständig zunehmenden Menge von Teilen. Diese «Grundsätzlichkeiten» sind vielfach mehr aus einer eigenständigen Evolution von wissenschaftlichen Fächern und Subspezialitäten hervorgegangen als aufgrund ihrer Bedeutung für Gesundheit und Krankheit oder für Kompetenz in der medizinischen Praxis. Die Gesamtheit des auf diese Weise zusammenge- 
tragenen Wissens ist für den individuellen Gebrauch und für die Lehre nicht mehr überblickbar noch organisierbar ${ }^{5}$. Sie muss heute mehr als optionale wissenschaftliche Ressource denn als Grundlage gesehen werden.

\section{Konsequenzen für das Gesundheitswesen}

Wenn isoliertes Fachwissen nicht länger als «grundlegend» bezeichnet werden kann, was ist in diesem Fall für die Medizin im allgemeinen und demzufolge für die medizinische Ausbildung grundlegend? Es wird in diesem abschliessenden Abschnitt um die Umsetzung eines integrierten biosemiotischen Denkmodells gehen. Dies sollte eigentlich nicht als Paradigmenwechsel, sondern als Erweiterung, Aufstockung einer rationalistisch-materialistischen Philosophie der Aufklärung bezeichnet werden [27]. Die letztere bleibt als Element unserer westlichen Kultur weiterhin unverzichtbar. Ein fundamentalistisches Festhalten und «Reinhalten» an dieser heute als reduktionistisch zu bezeichnenden Philosophie hat jedoch zu ernsthaften Problemen in unserer Gesellschaft geführt. Trotzdem stossen die hier diskutierten Erweiterungen auf einen massiven Widerstand, im Gesundheitsbereich vor allem von seiten industriell-gewinnorientierter und elitär-professioneller Gruppen und Institutionen [28]. Ohne auf diese wissenschafts- und bildungspolitische Pattsituation zusätzlich einzugehen, wollen wir hier exemplarisch einige ausgewählte Ansätze von Reorientierung und Reform skizzieren. Sie betreffen eine kritische Sicht auf Forschungsinhalte sowie zusätzliche Ansprüche an die ärztliche Kompetenz und entsprechende Reformen der medizinischen Ausbildung und der akademischen Hierarchie.

\section{Forschungsinhalte}

Im heute die Forschung dominierenden akademischen System bleiben viele Fragen zum Verständnis des Panoramas von Gesundheit und Krankheit unbeantwortet. Es sind dafür Studien zu entwerfen, Instrumente zu entwickeln, Daten $\mathrm{zu}$ erheben und brauchbare Information $\mathrm{zu}$ beschaffen. Empirische Forschung durch Allgemeinärztinnen und andere Generalisten, vorwiegend im ambulanten und hausärztlichen Versorgungsbereich, muss gegenüber den etablierten «akademischen» Schwerpunkten intensiviert werden. Die folgenden Beispiele haben die Analyse der Kausalitätsnetze zum Ziel, von denen wir behaupten, dass sie mit den Manifestationen von Gesundheit und Krankheit verbunden sind [29]:
- Welche Rolle spielen soziokulturelle Einstellungen bezüglich einer Entstellung der Brust für den Verlauf und bezüglich der Malignität von Brustkrebs [30]?

- Welchen Einfluss hat die Trennung von einem Familienmitglied, von einem Nachbarn oder selbst von einem Haustier auf die Progredienz von Herzinsuffizienz [31]?

- Welche Mortalitätsstatistiken korrelieren mit dem Zugang oder Nichtzugang zu unterstützenden sozialen Netzen in der unmittelbaren Umgebung von Individuen [32]?

- Wie korreliert die psychosoziale Situation von Berufstätigen mit der Häufigkeit von Myokardinfarkten [33, 34]?

- Ist der Plazeboeffekt der Ausdruck einer Illusion (ein Artefakt, wie er oft genannt wird), einer statistischen Streuung oder des natürlichen Verlaufes der Krankheit [35]?

- Korreliert der «Heilungskontext» oder die «Heilungsbeziehung» mit der Verbesserung des somatischen Zustandes direkt oder durch Potenzierung konventioneller oder «alternativer» Behandlungsverfahren [36-39]?

- Wie beeinflussen Ängste, Bedrohungen, Kummer und Trauer enger Verwandter die Morbidität und die Mortalität [40, 41]?

- Ist Depression, wenn sie mit bestimmten Zuständen verbunden ist, zum Beispiel mit Pneumonie, ein Vorläufer oder eine Folge der Krankheit?

- Warum erholen sich Kranke in gewissen Spitälern schneller als in anderen [42, 43]?

- Gibt es eine Beziehung zwischen emotioneller und spiritueller Entwicklung und dem Wachstum von Krebs oder der Überlebenszeit?

\section{Ärztliche Kompetenz: von der Beobachtung zur Interaktion}

Die Bedeutung der Beziehung zwischen Subjekt und Objekt lässt sich aus der Abb. 1 ablesen. Die Partnerin oder der Partner in jeder Beziehung ist immer Teil der «Umwelt» der anderen Person (Beispiele Individuum-Mutter und IndividuumArzt in der Abb. 2). Im ärztlichen Gespräch muss das Ziel die Schaffung der bereits erwähnten gemeinsamen Wirklichkeit zwischen Ärztinnen und Patienten sein. Interaktion muss als ein Akt zielgerichteten und wissenschaftlich begründeten Handelns betrachtet werden und nicht lediglich Ausdruck einer humanistischen Haltung oder einer Bemühung, «mit Patienten freundlicher zu sein».

Selbstverständlich dürfen traditionelle, biomechanisch orientierte Ärzte nicht beschuldigt werden, inhuman zu sein. Falls unsere Analyse gültig ist, muss aber die Forderung aufgestellt 
werden, dass die medizinischen Fakultäten auf der konzeptionellen und praktischen Ebene eine integrierende Sicht der Umwelt vermitteln, welche lebende Systeme, einschliesslich Patienten, als ihre eigene ansehen. Konzeptionell heisst, im Rahmen einer Erkenntnis- und Wissenschaftstheorie über «grundlagenwissenschaftliche» («Grundlage» in einem neuen Sinn, s. oben) Einsichten in die systemischen und biosemiotischen Aspekte von Lebensprozessen zu verfügen. Auf dieser Grundlage erhält eine schon seit einiger Zeit in der Ausbildung geforderte und geförderte praktische Kompetenz einen zusätzlichen Stellenwert: Die Fähigkeit zuzuhören, zu beobachten, Informationen einzuholen und deren Zuverlässigkeit, Gültigkeit und Qualität zu beurteilen [44].

Die Art der Kontrolle, welche Ärzte in traditionellen biomechanischen klinischen Beziehungssystemen ausübten, schützte sie vor einigen sehr beunruhigenden Gefühlen. Diese Kontrolle mit den Patienten zu teilen, auf Macht $\mathrm{zu}$ verzichten und die Formulierung von Gefühlen zu fördern, entfernt diese Schutzmauer. Viel mehr Verständnis für gegenseitige Beziehungen und, vor allem, Selbstkenntnis sind erforderlich [45].

\section{Kritische Sicht auf den medizinischen Fortschritt}

Eine epidemiologische Sicht der Medizingeschichte müsste die Beurteilung von Auswirkungen klinischer Interventionen auf die Gesundheit der Bevölkerung in einen weiteren Rahmen stellen. So wird unter anderem die Zunahme der Lebenserwartung oft als Konsequenz der Behandlung von Infektionskrankheiten mit Antibiotika angesehen. Thomas McKeowns (1913-1988) gründliche Analyse von historischen Gegebenheiten [46] hat gezeigt, dass «medizinische» Faktoren oft eine relativ geringe Rolle gespielt haben. Während des 19. und 20. Jahrhunderts hat beispielsweise die tuberkulosebedingte Mortalität in westlichen Gesellschaften stetig abgenommen. Die Einführung von spezifischen Tuberkulostatika ist in der Mitte des vergangenen Jahrhunderts mit einer lediglich minimalen Beschleunigung dieser Tendenz verbunden gewesen. Andere Faktoren wie die Verbesserung der Ernährung, der Wasserversorgung und der sanitären Einrichtungen scheinen viel wichtiger gewesen zu sein. Das Wiederauftreten von Armut, Verfremdung und ungenügender Ernährung kann sehr wohl mit heutigen Zunahmen von Tuberkuloseinzidenz verbunden werden [46]. Ein analoger Umstand führt heute im Falle von AIDS in Afrika zu einer vergleichsweise noch grösseren Katastrophe, wenn ein von der Industrie vorangetriebenes Forschungsmodell und politischer Druck prioritär auf die Beschaffung von Medikamenten anstatt auf die Sanierung von Defiziten in den Bereichen Ernährung und Hygiene orientiert ist [47]. Diese Beispiele illustrieren die ständige Herausforderung an die ärztliche «Kontext-Sensitivität». Ärzte und Ärztinnen sind gefordert «gross zu denken», um dann möglicherweise «klein zu handeln», dabei u.U. auf aufwendige pharmakologische oder technische Behandlungsformen zu verzichten.

Dieser historischen Einsicht sind Hinweise aus dem Bereich der klinischen Epidemiologie hinzuzufügen. So erweist sich die Auswirkung der Frühdiagnose von Brustkrebs mittels Mammographie-Massenscreening als zweifelhaft. Statistische Erhebungen an Gruppen von vergleichbaren Probandinnen ergeben bilanzmässig keine vorteilhafteren Verläufe [48]. Der Tendenz von «Frühdiagnosen» mit entsprechenden Frühinterventionen muss eine generelle «Ökonomie des Belebten» bzw. eine «mentation of nature» [49] entgegengesetzt werden: eine Fähigkeit lebender Systeme, mit phänomenologisch stummen Schädigungen «umzugehen». Ebenso interessant ist die Erkenntnis, dass traumatochirurgische Eingriffe an gewissen Bänderrissen gegenüber einem konservativen Vorgehen langfristig keine Vorteile ergeben [50]. Eine gezielte Forschung anhand von derartigen alternativen Behandlungsmaximen könnte sich gesundheitsökonomisch als äusserst interessant erweisen.

Aus diesen Entwicklungen ergeben sich systemische, für die medizinische und soziale Versorgung entscheidende Sichtweisen und Konzepte. Sie müssten bedeutende Implikationen für die ärztliche Ausbildungspolitik und Berufsentwicklung haben.

\section{Die medizinische Ausbildung}

Ausbildungsreformen haben sich bis heute vorwiegend auf pädagogische und didaktische Aspekte, etwa die Einführung von «problemorientiertem Lernen» anstelle von Frontalunterricht, beschränkt. Sie müssten aber nicht nur Antworten auf die Frage des Wie, sondern auch und vor allem nach dem Was der Ausbildung finden. Damit würde die Basis einer Wissenschaft und Forschung einer neuen Generation gelegt als Voraussetzung einer Versorgung im Gesundheitsbereich, die den Anforderungen der $\mathrm{Zu}$ kunft dienen könnte. Daraus ergibt sich beispielsweise die Vision eines medizinischen Ausbildungscurriculums. Warum sollen Studierende nicht in die Medizin auf ihrer echt grundlegenden Ebene eingeführt werden, d.h. anhand eines 
somatopsychosoziokulturellen Modells, kombiniert mit einer frühen Interaktion mit Personen/ Patienten? Damit würden sie mit Methoden systemischer Wissenschaften vertraut, die sich mit den Interaktionen von Individuen und deren Umgebung beispielsweise im Bereiche der Neurowissenschaften, Immunologie, Epidemiologie, Psychologie und Soziologie befassen. In einem derartigen Modell würden die bisherigen «Grundlagenwissenschaften» im Kontext des neuzeitlichen problemorientierten Lernens zu Instrumentalwissenschaften, die systemischen Anteile müssen dann als grundlegend betrachtet werden [3, 51]. Ausbildungsziel wäre eine erhöhte ärztliche Fähigkeit und Bereitschaft, auf viele Beobachtungen und Botschaften einzugehen, die immer wieder durch die Weltanschauung, welche unserer westlichen Medizin zugrundeliegt, ausgeklammert worden sind.

\section{Eine basisorientierte Versorgung}

Ein mehr generalistisch orientiertes Versorgungssystem erfordert eine qualitativ und quantitativ stärkere Beteiligung der in der Grundversorgung Tätigen an Politik und Steuerung in Lehre, Versorgung und Forschung [52]. Dies müsste mit einer beträchtlichen Aufstockung der ihnen zur Verfügung stehenden Ressourcen und Lerngelegenheiten einhergehen. Die Natur der schwergewichtig hoch spezialisierten akademischen Elite muss hinterfragt werden. Heute ist diese eng verbunden mit der «Prädominanz der Teile» (siehe oben). Nobelpreisträger in der Medizin sind fast ausschliesslich Molekularbiologen oder Genetiker. Hoch spezialisierte fachorientierte Forschung wird weiter benötigt sein, sollte aber die Ressourcen mit einer interdisziplinären Gesundheitsforschung (s. oben, Forschungsinhalte) teilen. Insbesondere muss ein neues Paradigma die Politik, die Planung und die Organisation des Gesundheitswesens und der sozialen Versorgung bestimmen. Systemisches und integrierendes Denken muss denjenigen, die grundlegende Beschlüsse fassen, zur Verminderung eines grossen Teiles der Ungleichheit, der Ineffizienz und der Vergeudung im Bereich der heutigen westlichen Gesundheitssysteme dienen. Innerhalb der Institutionen der Versorgung muss die Natur der Beziehungen zwischen Ärzten und Angehörigen anderer Gesundheitsberufe, speziell der Pflege, sowie Managerinnen und Ökonomen grundsätzlich diskutiert werden [53].

Während Berufsangehörige in eng umschriebenen Bereichen eher frei sind in der Entscheidung für extrem spezialisierte oder mehr generalistische Interessen, haben Ärztinnen mit Ver- antwortung für die Grundversorgung keine derartige Freiheit der Wahl. Es ist gleichzeitig dieses Ausmass an Verantwortung, repräsentiert durch Familien- und andere grundversorgende Ärzte und Ärztinnen, das den Inhalt der medizinischen Ausbildung bestimmen sollte. Das sich nur langsam entwickelnde Kader von konzeptmässig kompetenten generalistischen Praktikerinnen und Praktikern (u.a. Familienärztinnen, allgemeine Internisten, Pädiaterinnen und Psychiater) in Zusammenarbeit mit Vertretern anderer grösserer Gesundheitsberufe, wie derjenigen, die sich mit Pflege und «Public Health» befassen, wären dazu aufgerufen. Insbesondere vom Pflegebereich, dessen Ausbildungssysteme in bezug auf spezifisch humane Anteile denjenigen der ärztlichen Ausbildung überlegen sind, wären entsprechende Impulse zu erwarten. Spezialisierte Fachbereiche würden ihre Dienstleistungen dort anbieten, wo sie benötigt werden: eine Umkehr der Hierarchien in der medizinischen Wissenschaft!

\section{Literatur}

1 Regenbogen A, Meyer U (Hrsg.). Wörterbuch der philosophischen Begriffe. Hamburg: Felix Meiner Verlag; 1998.

2 von Uexküll J, Kriszat G. Streifzüge durch die Umwelten von Tieren und Menschen. Frankfurt a.M.: Fischer; 1970.

3 von Uexküll T, Wesiack W. Theorie der Humanmedizin: Grundlagen des ärztlichen Denkens. München, Wien, Baltimore: Urban \& Schwarzenberg; 1998.

4 Kuhn TS. Gespräch mit H. G. Pauli. Zürich, 1986.

5 Foss L. The End of Modern Medicine. Biomedical Science under a microscope. New York: State University of New York Press; 2002.

6 Kornberg A. The two cultures: chemistry and biology. Biochemistry 1987;26:6888-91.

7 World Health Organization. International Classification of Diseases. $10^{\text {th }}$ revision. Geneva: WHO; 1994.

8 Meier-Seethaler C. Gefühl und Urteilskraft. Ein Plädoyer für die emotionale Vernunft. München: Beck; 1997.

9 Bohr N. Atomphysik und Naturbeschreibung. Berlin: Springer; 1931.

10 von Bertalanffy L. General Systems Theory. Foundations, Development, Applications. New York: Braziller; 1968.

11 Prigogine I, Stengers I. Order out of Chaos: Man's New Dialogue with Nature. Glasgow: Collins; 1984.

12 Fleck L. Entstehung und Entwicklung einer wissenschaftlichen Tatsache. Frankfurt: Suhrkamp Taschenbuch Wissenschaft 312; 1980. 
13 Maturana H, Varela F. Der Baum der Erkenntnis. Wie wir die Welt durch unsere Wahrnehmung erschaffen - die biologischen Wurzeln des menschlichen Erkennens. Bern: Scherz; 1987.

14 Jantsch E. Die Selbstorganisation des Universums. München: Deutscher Taschenbuch Verlag; 1982.

15 McClintock B. The significance of responses of the genome to challenge. Science 1984; 226:702-801.

16 Strohman RL. The coming Kuhnian revolution in biology. Nature Biotechnology 1997;15:194-200.

17 Venter JC, Adams MD, Myers EW, Li PW, Mural RJ, Sutton GG, et al. The sequence of the human genome. Science 2001;291(5507):1304-51.

18 von Uexküll T, Pauli HG. The mind-body problem in medicine. Advances 1986;3:158-74.

19 Ulrich G. Biomedizin. Die folgenschweren Wandlungen des Biologiebegriffs. Stuttgart \& New York: Schattauer, 1997.

20 Foss L, Rothenberg K. The Second Medical Revolution. From Biomedicine to Infomedicine. Boston: New Science Library; 1987.

21 Burger W. Existiert eine unüberbrückbare Kluft zwischen Phänomenologie und Reduktionismus? In: Collegium Helveticum (Hrsg.). SternwartenBuch. Zürich: Haffmanns Sachbuch; 1999. S. 137-163.

22 von Uexküll J. Bedeutungslehre. Frankfurt a.M.: Fischer; 1970.

23 Antonovsky A. Unraveling the Mystery of Health. How People Manage Stress and Stay Well. San Francisco: Jossey-Bass; 1987.

24 Margraf, J, Siegrist J, Neumer S. Gesundheitsoder Krankheitstheorie? Berlin \& Heidelberg: Springer; 1998.

25 Trenkel A. Das Phänomen der Beziehung in der therapeutischen und pflegerischen Praxis. Gruppenpsychotherapie und Gruppendynamik 1997;33:243-58.

26 McWhinney IR. The importance of being different. Br J Gen Pract 1996;46:433-6.

27 Kuhn TS. Die Entstehung des Neuen. Frankfurt: Suhrkamp Taschenbuch Wissenschaft 236; 1978.

28 Bloom SW. Structure and ideology in medical education. An analysis of resistance to change. J Health Soc Behav 1988;29:294-306.

29 MacMahon B, Pough TF. Epidemiology: Principles and Methods. Boston: Little, Brown; 1970.

30 Edelstyn GA, McRae KD. Breast cancer: mistaken concepts, therapeutic consequences, and future implications. J Irish Med Assoc 1975;68:30-2.

31 White KL, Martin DA, Vernon CR. Venous pressure, emotions, and congestive heart failure. J Chro Dis 1959;10:163-85.

32 Berkman LF, Syme SL. Social networks, host resistance, and mortality: a nine year follow-up study of Alameda County residents. Amer J Epidemiol 1979;109:186-204.

33 Marmot MG, Bosma H, Hemigway H, Brunner E, Stansfeld S. Contribution of job control and other risk factors to social variations in coronary heart disease incidence. Lancet 1997;350:235-9.
34 Siegrist J, Matschinger H, Cremer P, Seidel D. Atherogenic risk in men suffering from occupational stress. Atherosclerosis 1988; 69:211-8.

35 Ernst E, Resch KL. Concept of true or perceived placebo effects. Br Med J 1995;311:551-3.

36 Brody H. The Healers Power. New Haven \& London: Yale University Press; 1982.

37 Harrington A (ed.). The Placebo Effect: An Interdisciplinary Exploration. Cambridge, MA \& London: Harvard University Press; 1997.

38 Schönbächler G. Placebo from a biosemiotic point of view. Forsch Komplementarmed 1989;5:18-23.

39 Spiro HM. The Power of Hope: a Doctor's Perspective. New Haven \& London: Yale University Press; 1998.

40 Cannon WB. «Voodoo Death». Psychosom Med 1982;19:182-90.

41 Kiecolt-Glaser JK. Psychoneuroimmunology and health consequences: data and shared mechanisms. Psychosom Med 1995;124:296-7.

42 Gillespie R. Manufacturing Knowledge: A History of the Hawthorne Experiments. Cambridge \& New York: Cambridge University Press; 1991.

43 Revans RW. The Hospital as a Human System. Bull N Y Acad Med 1996;73:418-29.

44 White KL. The Task of Medicine. Dialogue at Wickenburg. Menlo Park, CA: Kaiser Family Foundation; 1988.

45 Lipkin M Jr, Putnam SM, Lazare A (ed.). The Medical Interview: Clinical Care, Education, and Research. New York, Berlin: Springer; 1995.

46 McKeown T. The Role of Medicine. Dream, Mirage, or Nemesis? London: The Nuffield Provincial Hospitals Trust; 1976.

47 Root-Bernstein R. Rethinking AIDS. The Tragic Cost of Premature Consensus. New York: The Free Press/Macmillan; 1993.

48 Schmidt JG, Steele ES. Kritik der medizinischen Vernunft. Mainz: Kirchheim; 1997.

49 Bateson G. Mind and Nature. A Necessary Unity. Glasgow: Fontana; 1980.

50 Dubs L. Die Kunst der Unterlassung. Unsorgfältige Studien fördern eine Maximalmedizin. Neue Zürcher Zeitung 26.3.2002.

51 Pauli HG, Schüffel W. Wandel des Denkens in der Medizin? In: Schüffel W, Brucks U, Johnen J, Köliner V, Lamprecht F, Schnyder U (Hrsg.). Handbuch der Salutogenese: Konzept und Praxis. Wiesbaden: Ullstein Medical; 1998. S. 245-259.

52 McWhinney IR. Family medicine as a science. J Fam Pract 1978; 7:53-8.

53 White KL. Healing the Schism: Epidemiology, Medicine, and the Public's Health. New York and Heidelberg: Springer; 1991. 